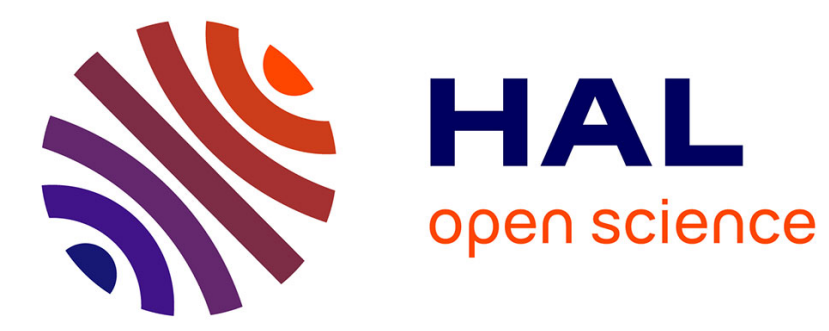

\title{
Efficient Bragg-like operation of intracavity low-efficiency plane gratings
}

Aurélie Moreau, Isabelle Zaquine, Alain Maruani, Robert Frey

\section{To cite this version:}

Aurélie Moreau, Isabelle Zaquine, Alain Maruani, Robert Frey. Efficient Bragg-like operation of intracavity low-efficiency plane gratings. Journal of the Optical Society of America B, 2005, 22 (11), pp.2289. hal-00862293

\section{HAL Id: hal-00862293 \\ https://hal-iogs.archives-ouvertes.fr/hal-00862293}

Submitted on 16 Sep 2013

HAL is a multi-disciplinary open access archive for the deposit and dissemination of scientific research documents, whether they are published or not. The documents may come from teaching and research institutions in France or abroad, or from public or private research centers.
L'archive ouverte pluridisciplinaire HAL, est destinée au dépôt et à la diffusion de documents scientifiques de niveau recherche, publiés ou non, émanant des établissements d'enseignement et de recherche français ou étrangers, des laboratoires publics ou privés. 


\title{
Efficient Bragg-like operation of intracavity low-efficiency plane gratings
}

\author{
Aurélie Moreau, Isabelle Zaquine, and Alain Maruani \\ Département Traitement du Signal et de l'Image, Ecole Nationale Supérieure des Télécommunications, 46, rue \\ Barrault, 75634 Paris Cedex 13, France
}

\begin{abstract}
Robert Frey
Département Traitement du Signal et de l'Image, Ecole Nationale Supérieure des Télécommunications, 46, rue Barrault, 75634 PARIS cedex 13, France and Laboratoire Charles Fabry de l'Institut d'Optique, Centre Scientifique de Paris Sud Bât 503, 91403 Orsay Cedex, France
\end{abstract}

Received February 18, 2005; revised manuscript received May 19, 2005; accepted May 23, 2005

\begin{abstract}
The diffraction regime of a thin grating inserted in a Fabry-Perot cavity is investigated. Our calculations show that, at Bragg incidence, a single diffraction order can be selectively enhanced, giving rise to a very efficient Bragg-like diffraction regime. The optimization of the device is studied as a function of the resonator thickness and finesse and the grating position inside the Fabry-Perot cavity. The angular and wavelength selectivities are also investigated. The device could be easily integrated and would be very useful for optical signalprocessing applications. (c) 2005 Optical Society of America
\end{abstract}

OCIS codes: $050.1970,050.1950,050.2230$.

\section{INTRODUCTION}

Diffraction efficiency increase is a very important issue in optical signal processing. ${ }^{1-3}$ The Bragg diffraction regime, ${ }^{4}$ in which the entire diffracted energy is concentrated in a single diffraction order, is the most efficient regime. This regime can be achieved, at Bragg incidence, only beyond some grating thickness threshold. It has been shown that inserting a volume grating in a Fabry-Perot resonator is a good means of improving its efficiency, especially if the volume grating fills the cavity entirely. ${ }^{5}$ In that case, the thickness threshold toward the Bragg regime can be significantly lowered ${ }^{6}$ because of the multiple paths in the nonlinear medium, but many efficient nonlinear media are available only in such small thicknesses that no Bragg regime can be obtained in this way.

Here, we investigate another diffraction regime occurring when a thin grating is inserted in a Fabry-Perot resonator. Most of the time, such a device is operated at normal incidence to avoid the walk-off of the read beam in the Fabry-Perot cavity. ${ }^{7-13}$ In that case, only the read beam is Fabry-Perot resonant, and the diffracted beams are all enhanced in the same manner. To favor a unique diffraction mode, we use Bragg incidence and tune the resonator accordingly. Both read and first-order diffracted beams are then Fabry-Perot resonant, whereas higherorder diffraction modes are not. This results in a selective enhancement that can lead, as we will show, to a diffraction regime in which the intensity of higher diffraction orders is at least 100 times smaller than that of the main diffraction order. This can usually be obtained only with volume gratings or Bragg gratings, and we call it a "Bragg-like" diffraction regime.

Section 2 describes the model used for the calculations. Section 3 shows how the cavity thickness, the reflection coefficient, the one-pass grating efficiency, and the position of the grating in the cavity can be chosen in order to obtain a Bragg-like diffraction regime and to optimize the diffraction efficiency. Finally, Section 4 deals with the angular and wavelength selectivities of the device.

\section{MODEL}

The unslanted sinusoidal refractive-index grating, of intensity diffraction efficiency $\rho_{g}$, is inserted between two mirrors with reflection coefficients $R_{1}$ and $R_{2} \approx 1$, respectively [see Fig. 1(a)]. The thickness of this asymmetric Fabry-Perot cavity is $L$. The real refractive index of the cavity medium is $n$. When illuminated by a plane wave of wavelength $\lambda_{0}$ and incidence angle $\theta_{0}$, the grating gives rise to a transmitted beam (order 0 ) and two diffraction orders -1 and +1 .

The propagation angle $\theta_{p}=\left(\mathbf{z}, \mathbf{k}_{\mathbf{p}}^{\mathbf{F}, \mathbf{B}}\right)$ of the $p$ th order forward- and backward-diffracted waves (of wave vectors $\mathbf{k}_{\mathbf{p}}^{\mathbf{F}}$ and $\mathbf{k}_{\mathbf{p}}^{\mathbf{B}}$, respectively) in the intracavity medium is determined by the Raman-Nath relation: $k\left(\sin \theta_{p}-\sin \theta_{0}\right)$ $=p K$, where $k=2 \pi n / \lambda_{0}$ and $K=2 \pi / \Lambda$ ( $\Lambda$ is the spatial period of the grating). The corresponding forward and backward wave vectors $\mathbf{k}_{\mathbf{p}}^{\mathbf{F}, \mathbf{B}}$ are shown in Fig. 1(b).

When the read beam is set at Bragg incidence $\theta_{B}\left(\theta_{0}\right.$ $\left.=\theta_{B}\right)$, the diffraction order -1 is symmetrical to the read beam with respect to the $z$ axis [see Fig. 1(b)]. The FabryPerot cavity can then be tuned to both read and diffraction-order -1 beams. Considering that order +1 is generally not Fabry-Perot resonant in the device, for the sake of simplicity, the waves of order $p$ greater than one are neglected and only the higher order -2 , resulting from 


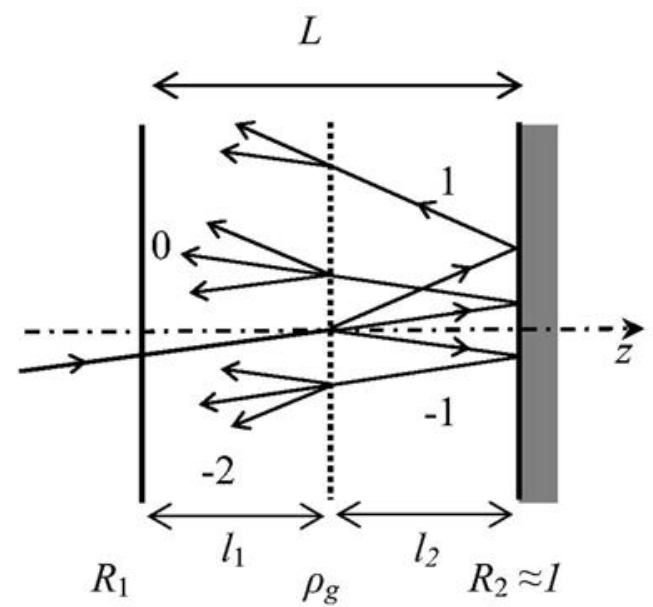

(a)

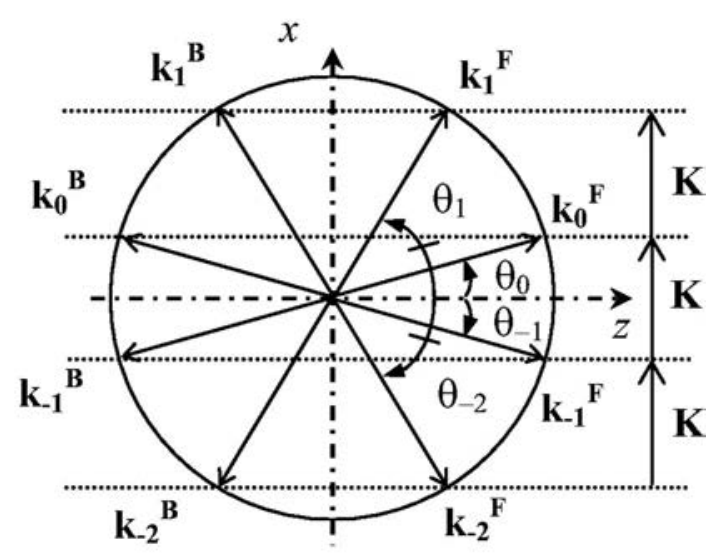

(b)

Fig. 1. (a) Setup of the thin grating inside the Fabry-Perot resonator and (b) the wave vectors of all considered diffraction orders.

the diffraction of the resonant order -1 and symmetrical to the order +1 with respect to the $z$ axis, is taken into account.

The reflected diffracted amplitudes of orders $0,-1,+1$, and -2 are calculated using the product of matrices describing reflection on the mirrors, propagation in the cavity, and diffraction on the thin grating, using a standard technique used for the description of multilayered dielectric media. $^{14}$
A four-dimensional vector $\mathbf{F}$ (or $\mathbf{B}$ ) is formed with the four forward- (or backward-) propagating complex amplitudes $S_{p}$ corresponding to the diffraction orders from $p=$ -2 to $p=+1$.

$$
\mathbf{F}(\operatorname{resp} \mathbf{B})=\left(\begin{array}{c}
S_{-2} \\
S_{-1} \\
S_{0} \\
S_{+1}
\end{array}\right)_{\mathbf{F}(\operatorname{resp} \mathbf{B})} \text {. }
$$

An eight-dimensional vector $\operatorname{col}(\mathbf{F}, \mathbf{B})$ is formed with all amplitudes. Beam amplitudes considered before and after the device are labeled using subscripts bd and ad, respectively.

Considering the device of Fig. 1(a), the beam amplitudes before and after the device are related by

$$
\left(\begin{array}{l}
\mathbf{F}_{\mathrm{bd}} \\
\mathbf{B}_{\mathrm{bd}}
\end{array}\right)=M_{1} P_{1} D P_{2} M_{2}\left(\begin{array}{l}
\mathbf{F}_{\mathrm{ad}} \\
\mathbf{B}_{\mathrm{ad}}
\end{array}\right),
$$

where $M_{1}$ and $M_{2}$ describe the reflection on the front and back mirrors, respectively; $P_{1}$ and $P_{2}$ are the propagation between the front mirror and the grating and that between the grating and the back mirror, respectively; and $D$ is the diffraction of the plane grating inserted in the cavity. $M_{1}, M_{2}, P_{1}, P_{2}$, and $D$ are all $8 \times 8$ matrices described hereafter as:

$$
M_{i}=\frac{1}{t_{i}}\left(\begin{array}{cc}
I_{4} & -r_{i} I_{4} \\
-r_{i} I_{4} & I_{4}
\end{array}\right) \quad \text { for } i=(1,2)
$$

where $I_{4}$ is the four-dimensional identity matrix; and $r_{i}$ and $t_{i}$ are, respectively, the amplitude reflection and transmission coefficients of the considered cavity mirror. $r_{i}$ is related to $R_{i}$ by $\left|r_{i}\right|^{2}=R_{i}$.

The propagation matrices are diagonal; each diagonal term represents the phase change $\left(\mathbf{k}_{\mathbf{p}}^{\mathbf{F}, \mathbf{B}} \cdot \mathbf{z} l\right)$ for a beam of wave vector $\mathbf{k}_{\mathbf{p}}^{\mathbf{F}, \mathbf{B}}$ propagating on a distance $l$. The two cases considered here are the propagation between the front mirror and the grating $\left(l_{1}\right)$ and the propagation between the grating and the back mirror $\left(l_{2}\right)$. The resonator length $L$ is the sum of $l_{1}$ and $l_{2}$ :

$$
P_{i}=\left(\begin{array}{cc}
P_{i 4}^{*} & 0_{4} \\
O_{4} & P_{i 4}
\end{array}\right) \quad \text { for } i=(1,2),
$$

where $O_{4}$ is the four-dimensional zero matrix and $P_{i 4}$ is the propagation submatrix:

$$
P_{i 4}=\left(\begin{array}{cccc}
\exp \left(j \mathbf{k}_{-2} \cdot \mathbf{z} l_{i}\right) & 0 & 0 & 0 \\
0 & \exp \left(j \mathbf{k}_{-1} \cdot \mathbf{z} l_{i}\right) & 0 & 0 \\
0 & 0 & \exp \left(j \mathbf{k}_{0} \cdot \mathbf{z} l_{i}\right) & 0 \\
0 & 0 & 0 & \exp \left(j \mathbf{k}_{+1} \cdot \mathbf{z} l_{i}\right)
\end{array}\right)
$$


The matrix $D$ is built from the symmetric, tridiagonal diffraction submatrix $D_{4}$ :

$$
D=\left(\begin{array}{cc}
D_{4}^{-1} & 0_{4} \\
0_{4} & D_{4}
\end{array}\right) \text {, with } D_{4}=\left(\begin{array}{cccc}
t_{g} & d_{g} & 0 & 0 \\
d_{g} & t_{g} & d_{g} & 0 \\
0 & d_{g} & t_{g} & d_{g} \\
0 & 0 & d_{g} & t_{g}
\end{array}\right)
$$

The $D_{4}$ matrix relates the amplitudes before the grating to the amplitudes after the grating. The amplitude of order $p$ after the grating is the sum of three contributions: The first one is due to the transmission by the grating of the amplitude of the order $p$, and the others are due to the diffraction by the grating of the amplitudes of the orders $p+1$ and $p-1$. The amplitude transmission coefficient $t_{g}$ and the diffraction coefficient $d_{g}=j \rho_{g}^{1 / 2}$ of this sinusoidal grating are related by the equation $\left|t_{g}\right|^{2}+2\left|d_{g}\right|^{2}=1$, where $\rho_{g}$ is the intensity diffraction efficiency of the grating. As not all the diffracted beams are considered in our analysis (we have limited our investigation to four diffractionorder beams only), the diffraction matrix is not unitary.

The numerical calculations are performed using a simple MATLAB routine.

\section{DEVICE OPTIMIZATION}

This section is devoted to the optimization of the device in terms of diffraction efficiency and Bragg-like operation. We define the diffraction efficiency as the ratio of the reflected diffracted intensity to the input intensity of the read beam, and we use the notation $\rho_{D}$ when we consider the order -1 beam. The refractive index of the medium is $n=2.8$, and the wavelength $\lambda_{0}$ is set at $1 \mu \mathrm{m}$. The Bragg angle is related to the grating period by $\sin \left(\theta_{\mathrm{B}}\right)$ $=\lambda_{0} /(2 n \Lambda)$. The incidence is set at the Bragg angle $\left(\theta_{\mathrm{B}}\right.$ $=5^{\circ}$ for the $2 \mu \mathrm{m}$ grating period $\Lambda$ considered here as an example). Finally, the back mirror is almost totally reflective $\left(R_{2} \approx 1\right)$.

Figure 2 shows the variation of the diffracted intensities as a function of the resonator length, where order 0 and order -1 are kept Fabry-Perot resonant (we consider only the resonator lengths for which order 0 and -1 are resonant). The thin grating of diffraction efficiency $\rho_{g}$ $=10^{-4}$ is positioned in the middle of the resonator, and the front-mirror reflectivity is $R_{1}=0.8$.

As orders 0 and -1 are always Fabry-Perot resonant, the diffraction efficiency is constantly equal to $\rho_{D}=10^{-1}$, showing a very large enhancement (by a factor of 1000) as compared with that of the bare grating. The diffraction efficiencies of orders +1 and -2 are lower than that of order -1 by more than $20 \mathrm{~dB}$, except for cavity lengths corresponding to quasi-resonance of these orders (i.e., when orders +1 and -2 are also close to another Fabry-Perot resonance) in the cavity. The closer that the +1 and -2 orders are to the Fabry-Perot resonance, the greater their diffraction efficiencies are. When the cavity is tuned to all considered diffraction orders, the diffraction efficiency of order +1 is almost as large as that of order -1 so that the diffraction efficiency of order -1 slightly decreases owing to the read-beam depletion by the order +1 beam. As shown in Fig. 2, this unfavorable situation can be easily avoided if the proper the resonator length is chosen. It must also be stressed that what we have called the Bragglike operation of plane gratings is possible with very thin devices that could be processed using nanotechnologies. However, for micrometer devices, the thickness of the grating must be taken into account, and the results presented here are no longer valid. Finally, although this result has almost no practical consequences, let us note that the decrease of orders +1 and -2 is an antiresonance owing to the sign change of the transmitted order 0 phase.

Figure 3 shows the influence of the reflection coefficient $R_{1}$ on the diffracted intensities for a cavity thickness $L$ $=3.55 \mu \mathrm{m}$, with the thin grating of diffraction efficiency $\rho_{g}=10^{-4}$ again set in the middle of the resonator. In Fig. $3(\mathrm{a})$, the diffraction efficiency of order -1 exhibits a maximum at $R_{1}=0.96$, which means that almost all the readbeam energy is transferred to the main diffraction order. This demonstrates the very high (10 000 in this case, for instance) increase in diffraction efficiency of the device. Naturally, the front-mirror reflectivity corresponding to the maximum diffraction efficiency increases when $\rho_{g}$ is decreased. Beyond this optimal value, the diffraction efficiency steeply decreases, owing to the fact that the energy

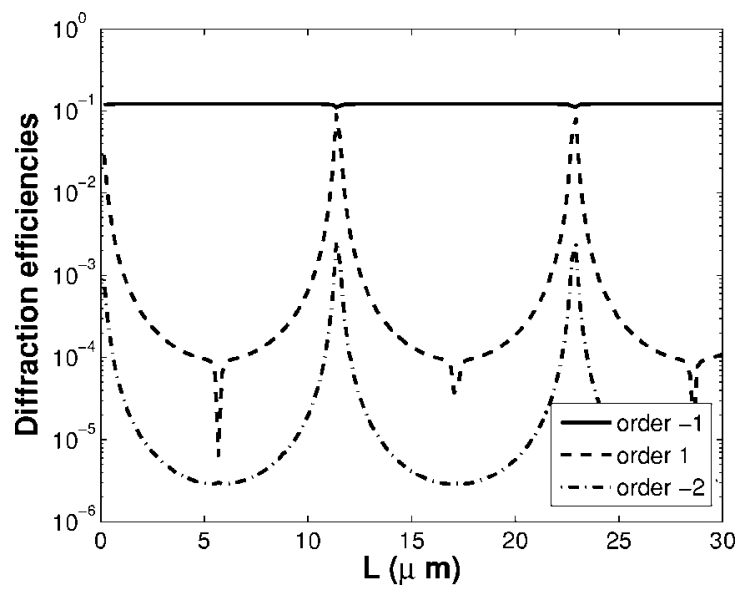

Fig. 2. Logarithmic plot of diffraction efficiencies as a function of resonator length with $\rho_{g}=10^{-4}$ and $R_{1}=0.8$.
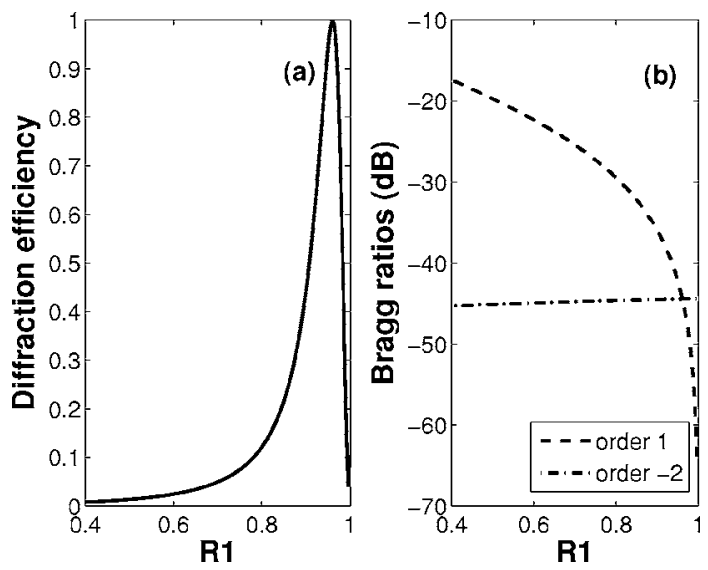

Fig. 3. (a) Diffraction efficiency of order -1 and (b) Bragg ratios as a function of the reflection coefficient of the first mirror, with $\rho_{g}=10^{-4}$ and $L=3.55 \mu \mathrm{m}$. 

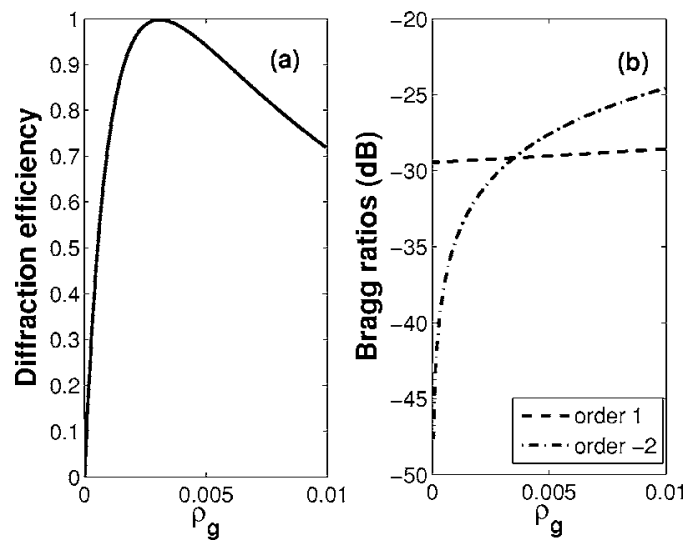

Fig. 4. (a) Order -1 diffraction efficiency and (b) Bragg ratios as a function of the one-pass efficiency of the grating with $L$ $=3.55 \mu \mathrm{m}$ and $R_{1}=0.8$.

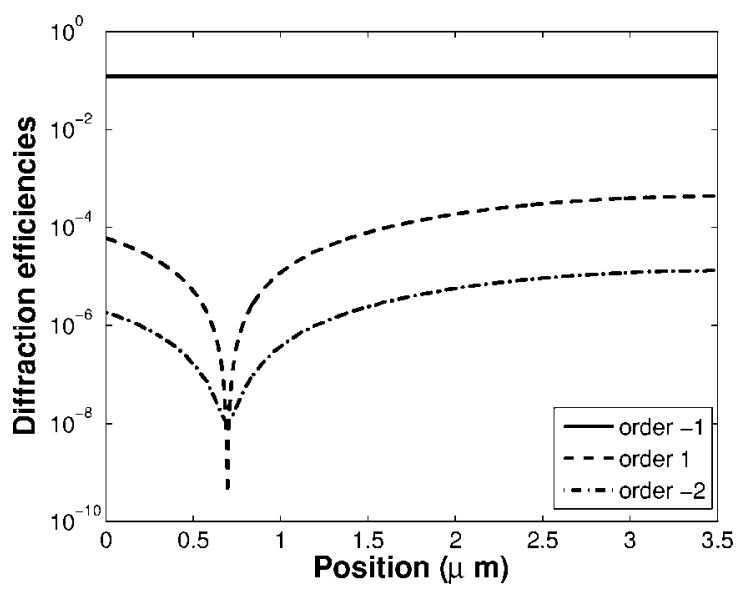

Fig. 5. Logarithmic plot of diffraction efficiencies as a function of the position of the grating inside the cavity with $\rho_{g}=10^{-4}, L$ $=3.55 \mu \mathrm{m}$, and $R_{1}=0.8$.

is diffracted back into order 0. The Bragg ratios shown in Fig. 3(b) represent the intensity ratio of the higher diffraction orders +1 and -2 to the Fabry-Perot resonant -1 diffraction order. For $R_{1}>0.51$ the Bragg ratio of order +1 is less than $-20 \mathrm{~dB}$, showing again that the actual Bragglike regime is easily obtained by inserting the plane grating in the Fabry-Perot cavity. Moreover, a very steep decrease of the Bragg ratio of this order with increasing Fabry-Perot finesse can be seen, owing to the good diffraction efficiency of order -1 . This decrease continues beyond $R_{1}=0.96$ because of the phase change of the laser reflected beam. Finally, the Bragg ratio of order -2 keeps almost constant at a very low value $(\approx-45 \mathrm{~dB})$, as the intensity of this mode is proportional to that of order -1 .

Figure 4 shows the variation of diffracted intensities as a function of the one-pass efficiency of the grating. The thin grating of diffraction efficiency $\rho_{g}$ is set in the middle of the $3.55 \mu \mathrm{m}$ long resonator, and the front-mirror reflectivity is $R_{1}=0.8$. The diffraction efficiency of the order -1 beam, shown in Fig. 4(a), exhibits a maximum at $\rho_{g}$ $=3.10^{-3}$, where all the read-beam energy is transferred to the main diffraction order as occurs when the resonator finesse is optimized [see Fig. 3(a)]. The Bragg ratios are represented in Fig. 4(b). The Bragg ratio of order +1 keeps almost constant on this logarithmic scale, owing to the fact that the energy of the 0 order beam is transferred in the same way to the order -1 beam and to the order +1 beam by the grating so that only the Fabry-Perot resonance makes the difference. The Bragg ratio of order -2 scales like $\rho_{g}$, because the order -2 originates only from the diffraction of order -1 . For the chosen values of $R_{1}$ and $R_{2}$, Bragg ratios are well below $-20 \mathrm{~dB}$, which means that a Bragg-like diffraction regime is easily obtained, whatever the one-pass efficiency of the thin grating.

Results shown in Figs. 2-4 correspond to a plane diffraction grating set in the middle of the cavity. Figure 5 shows the dependence of diffracted intensities as a function of the position of the grating in the cavity for a cavity thickness $L=3.55 \mu \mathrm{m}$, a reflection coefficient $R_{1}=0.8$, and a one-pass diffraction efficiency $\rho_{g}=10^{-4}$. As expected, the intensity of order -1 beam is constant. The sharp minimum of the higher orders is due to an antiresonance of these orders, owing to a phase discontinuity. The results shown in Fig. 5 demonstrate that at Bragg incidence, the position of the grating has almost no influence on the diffraction efficiency of order -1 or on the diffraction regime. This will simplify the fabrication of such devices. Let us mention only that, if necessary, the choice of the position of the grating could allow a much higher Bragg selectivity for the device. It must also be stressed that, owing to the low cavity thickness, the results presented here are valid only for very thin gratings (smaller than $100 \mathrm{~nm}$, obtained using quantum wells, for instance). However, the same results are obtained for thicker gratings, provided the cavity thickness is increased.

\section{SELECTIVITY}

In this section, we investigate angular and wavelength selectivities, which are important parameters for the application of any diffraction device. The thin grating $(2 \mu \mathrm{m}$ grating period) of diffraction efficiency $\rho_{g}=10^{-4}$ is set in the middle of the $3.55 \mu \mathrm{m}$ long resonator. The frontmirror reflectivity is $R_{1}=0.8$ and the back mirror is almost totally reflective.

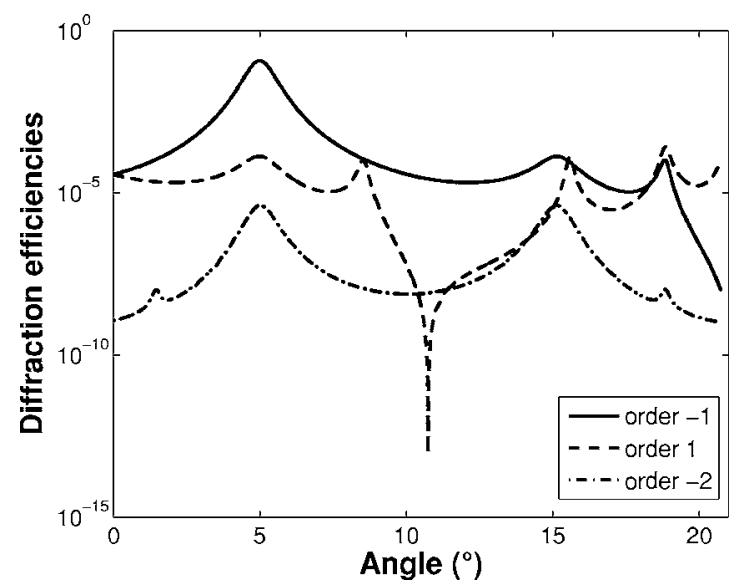

Fig. 6. Logarithmic plot of diffraction efficiencies as a function of incidence angle (inside the cavity) with $\rho_{g}=10^{-4}, L=3.55 \mu \mathrm{m}$, and $R_{1}=0.8$. 

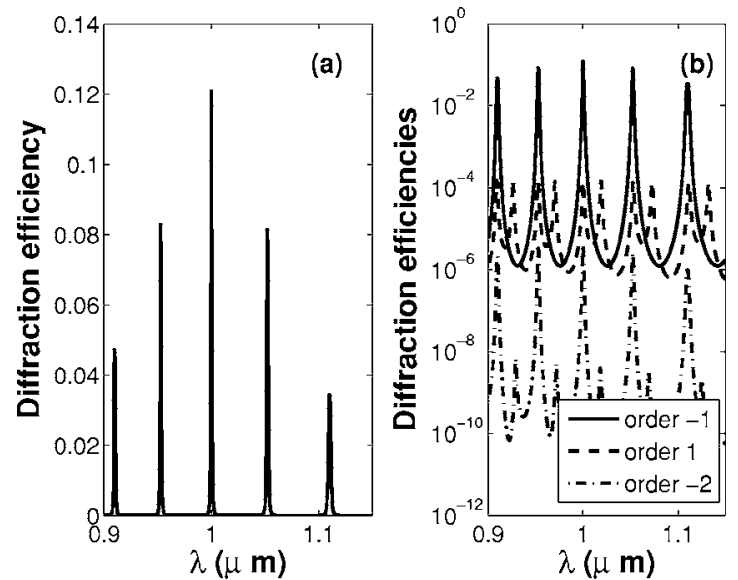

Fig. 7. (a) Order -1 diffraction efficiency and (b) Diffraction efficiencies plotted as a function of wavelength with $\rho_{g}=10^{-4}, L$ $=3.55 \mu \mathrm{m}$, and $R_{1}=0.8$.

The angular selectivity of the device was evaluated by calculating the diffracted intensities of orders $-1,+1$, and -2 as a function of the incidence angle of a laser beam of wavelength $\lambda_{0}=1 \mu \mathrm{m}$ inside the cavity (see Fig. 6). The incidence angle of the laser beam outside the cavity is varied from 0 to $\pi / 2$. Maxima correspond to resonances of the different beams. The Fabry-Perot is tuned to both read and diffraction order -1 beams only for $\theta_{0}=5^{\circ}$ (Bragg incidence for the $2 \mu \mathrm{m}$ grating period chosen here). As a consequence, the diffraction efficiency of the Bragg order -1 is maximum for this angle and decreases on both sides. The $3 \mathrm{~dB}$ width is $0.76^{\circ}$, which means that application to optical processing of complex signals such as correlation could be performed using such devices. Moreover, there is a $30 \mathrm{~dB}$ ratio between the order -1 beam and the higher orders at $\theta_{0}=5^{\circ}$. The maximum diffraction efficiency of order -1 beam exceeds that of higher diffraction orders at any angle by 20 or $30 \mathrm{~dB}$, which again demonstrates the Bragg-like regime of the device.

The wavelength selectivity of the device was evaluated by calculating the diffracted intensities as a function of the wavelength of the read beam for $\theta_{0}=5^{\circ}$ (see Fig. 7). When the read wavelength fulfills the Fabry-Perot resonance condition, the diffraction order -1 is also resonant, hence exhibiting periodic maxima of its intensity. In Fig. 7 (a) where the diffraction efficiency of order -1 is plotted on a linear scale, we can see a variation of the amplitude of the peaks due to the fact that the incidence angle is set at Bragg only for $\lambda_{0}=1 \mu \mathrm{m}$. In Fig. 7(b) a logarithmic scale has been chosen so that higher orders can be compared with the main diffraction order. Maxima of the higher-diffraction-order intensities are obtained on the one hand when the cavity is tuned to the read beam and on the other hand when it is tuned to the diffraction order +1 beam. The diffraction order -2 and +1 beams fulfill the same resonance condition because they have opposite angles. Because resonances for orders -1 and +1 never occur at the same wavelength, there is a $30 \mathrm{~dB}$ ratio between maxima of diffraction order -1 intensity and maxima of the higher-diffraction-order intensities. Multiwavelength efficient operation of the device is possible in the Bragg regime, which indicates that wavelengthmultiplexed Bragg-like plane gratings could also be used with this device.

\section{CONCLUSION}

Our calculations show that a Bragg-like diffraction regime can be obtained with a thin intracavity diffraction grating; the wavelength, incidence angle, and length must be chosen such that order 0 is simultaneously Fabry-Perot resonant and at Bragg incidence, so that diffraction order -1 is symmetrical to order 0 and is also Fabry-Perot resonant. We can notice that Bragg-like operation of plane gratings can be performed with micrometer cavities if the cavity length is not tuned to quasiresonance of higher orders. For a given $\rho_{g}$, the reflection coefficient of the front mirror can be chosen in order to maximize the diffraction efficiency of the device, and Bragg-like operation can be achieved, even with small diffraction efficiency of the grating. The grating position has no influence on the device performance, which makes the device easy to fabricate. Good angular selectivity has been demonstrated that could be useful for information processing. Multiwavelength efficient operation is possible as long as Fabry-Perot resonances are taken into account for the choice of the wavelengths. This device is a very promising one, as it combines very good performance and ease of integration using the state of the art photonics nanotechnologies.

A. Moreau's e-mail address is Aurelie.Moreau@enst.fr.

\section{REFERENCES}

1. L. Hesselink, S. S. Orlov, and M. C. Bashaw, "Holographic data storage systems," Proc. IEEE 92, 1231-1280 (2004).

2. J. Zhao, X. Shen, and Y. Xia, "Beam splitting, combining and cross coupling through multiple superimposed volume index gratings," Opt. Laser Technol. 33, 23-28 (2001).

3. Y. Ding, D. D. Nolte, M. R. Melloch, and A. M. Weiner, "Time domain image processing using dynamic holography," IEEE J. Sel. Top. Quantum Electron. 4, 332-340 (1998).

4. M. G. Moharam, T. K. Gaylord, and R. Magnusson, "Criteria for Bragg regime diffraction by phase gratings," Opt. Commun. 32, 14-18 (1980).

5. L. Menez, I. Zaquine, A. Maruani, and R. Frey, "Intracavity Bragg grating,” J. Opt. Soc. Am. B 16, 1849-1855 (1999).

6. L. Menez, I. Zaquine, A. Maruani, and R. Frey, "Bragg thickness criterion for intracavity diffraction grating," J. Opt. Soc. Am. B 19, 965-972 (2002).

7. D. D. Nolte, K. M. Kwolek, C. Lenox, and B. Streetman "Dynamic holography in a broad-area optically pumped vertical GaAs microcavity,” J. Opt. Soc. Am. B 18, 257-263 (2001).

8. A. Sinha and G. Barbastathis, "Resonant holography," Opt. Lett. 27, 385-387 (2002).

9. L. Escoubas, F. Flory, F. Lemarchand, A. During, and L. Roux, "Enhanced diffraction efficiency of gratings in multilayers," Opt. Lett. 25, 194-196 (2000).

10. C. De Matos, H. L'Haridon, A. Le Corre, R. Lever, J. C. Kéromnès, G. Ropars, C. Vaudry, B. Lambert, and M. Pugnet, "Epitaxial liftoff microcavities for $1.55 \mu \mathrm{m}$ 
quantum-well spatial light modulators," IEEE Photonics Technol. Lett. 11, 57-59 (1999).

11. R. Grac, M. Pugnet, J. H. Collet, B. Lambert, C. De Matos, H. L'Haridon, A. Le Corre, and J. O. White, "Photodiffraction in InGaAs/InGaAsP multiple quantum wells enclosed in a microcavity," Superlattices Microstruct. 22, 505-509 (1997).

12. K. Tian and G. Barbastathis, "Cross talk in resonant holographic memories," J. Opt. Soc. Am. A 21, 751-756 (2004).

13. D. D. Nolte and K. M. Kwolek, "Diffraction from a shortcavity Fabry-Pérot: application to photorefractive quantum wells," Opt. Commun. 115, 606-616 (1995)

14. M. Born and E. Wolf, Principle of Optics (Pergamon, 1970), p. 55 . 